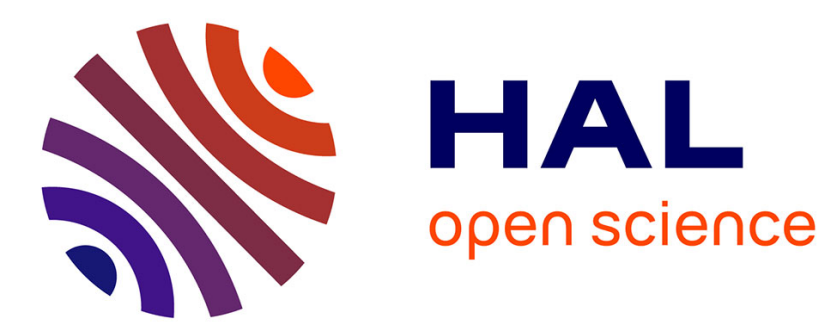

\title{
High-frequency driven capillary flows speed up the gas-liquid phase transition in zero-gravity conditions
}

Daniel Beysens, Denis Chatain, Pierre Evesque, Yves Garrabos

\section{To cite this version:}

Daniel Beysens, Denis Chatain, Pierre Evesque, Yves Garrabos. High-frequency driven capillary flows speed up the gas-liquid phase transition in zero-gravity conditions. Physical Review Letters, 2005, 95 (3), 034502 (4 p.). 10.1103/PhysRevLett.95.034502 . hal-00015420

\author{
HAL Id: hal-00015420 \\ https://hal.science/hal-00015420
}

Submitted on 1 Jul 2021

HAL is a multi-disciplinary open access archive for the deposit and dissemination of scientific research documents, whether they are published or not. The documents may come from teaching and research institutions in France or abroad, or from public or private research centers.
L'archive ouverte pluridisciplinaire HAL, est destinée au dépôt et à la diffusion de documents scientifiques de niveau recherche, publiés ou non, émanant des établissements d'enseignement et de recherche français ou étrangers, des laboratoires publics ou privés. 


\title{
High-Frequency Driven Capillary Flows Speed Up the Gas-Liquid Phase Transition in Zero-Gravity Conditions
}

\author{
Daniel Beysens, ${ }^{1}$ Denis Chatain, ${ }^{1}$ Pierre Evesque, ${ }^{2}$ and Yves Garrabos ${ }^{3}$ \\ ${ }^{1}$ ESEME, Service des Basses Températures, Commissariat à I'Energie Atomique, 17 rue des Martyrs, 38054 Grenoble Cedex 9, France \\ and Ecole Supérieure de Physique et Chimie Industrielle, 10 rue Vauquelin, 75231 Paris Cedex 05, France \\ ${ }^{2}$ Lab MSSMat, Ecole Centrale de Paris, UMR 8579 CNRS, 92295 Châtenay-Malabry Cedex, France \\ ${ }^{3}$ ESEME, Institut de Chimie de la Matière Condensée de Bordeaux, CNRS UPR 9048, \\ 87 avenue du Docteur A. Schweitzer, 33608 Pessac Cedex, France \\ (Received 7 January 2004; revised manuscript received 20 April 2005; published 14 July 2005)
}

\begin{abstract}
Under weightlessness conditions, the phase transition of fluids is driven only by slow capillary flows. We investigate the effect of high-frequency vibrations to reproduce some features of gravity effects and show that such vibrations can greatly modify the phase transition kinetics. The investigation is performed in $\mathrm{H}_{2}$ near its critical point (critical temperature $33 \mathrm{~K}$ ) where critical slowing down enables the phase transition process to be carefully studied. Gravity effects are compensated in a strong magnetic field gradient.
\end{abstract}

DOI: 10.1103/PhysRevLett.95.034502

The efficient management of fluids in space is necessary for the successful operation of spacecrafts and satellites. A near-zero-gravity environment results in fluids behaving in a manner markedly different from Earth-bound conditions. This demands a thorough understanding of a wide variety of fundamental processes. One of the most important processes is the gas-liquid phase transition. In space, the phase transition kinetics appears driven mainly by capillary flows and is strikingly slowed down as compared on Earth where buoyancy considerably speeds up the process [1]. In this Letter, we report on how high-frequency vibrations under weightlessness can modify and speed up phase transition, thus reproducing some features of gravity effects.

Vibrations that are considered here are linearly polarized with displacement $X=a \cos \omega t$ ( $a$ is the maximum amplitude, $\omega=2 \pi f$ is angular frequency, with $f$ the frequency). "High-frequency" vibrations are frequencies that are larger than the inverse of the typical hydrodynamic times. Such vibrations induce steady flow due to nonlinearities in the Navier-Stokes equations [2]. These nonlinearities are responsible, as is seen below, for considerable modification of the growth laws and pattern morphology in the course of the gas-liquid phase transition. Investigation of the mechanisms involved in these processes are facilitated by performing studies near the critical point in order to take advantage of universal scaling laws and critical slowing down of the dynamics [3]. Spacelike conditions are achieved by a strong magnetic field gradient that compensates the gravitational forces [4].

In a typical phase separation experiment the fluid is quenched from an initial state (at a temperature $T_{i}$ ) where it is homogeneous to another state (at a temperature $T_{f}$ ) below the coexistence curve where it is no longer thermodynamically stable. It has been found [1] that phase separation in gas-liquid systems (and liquid mixtures) obeys only two universal scaling laws of growth. The key parameter in these laws is the volume fraction $\phi$ of the
PACS numbers: 47.20. $-\mathrm{k}, 64.60 .-\mathrm{i}$, 64.70.Fx

minority phase $\left[\phi=v_{\mathrm{g}} /\left(v_{\mathrm{g}}+v_{\mathrm{l}}\right)\right.$, with $v_{\mathrm{g}, \mathrm{l}}$ the volume of gas (g) and liquid (1)] [1]. When $\phi<0.3$, growth is driven by droplet coalescences, which are controlled by Brownian motion. The typical evolution law is $L_{m} \sim t^{1 / 3}$ where $t$ is time and $L_{m}$ is the average distance between droplets. When $\phi>0.3$, the flow resulting from droplet coalescence is able to induce additional coalescence which eventually leads to an interconnected pattern with pseudowavelength $L_{m}$. Growth velocity $U$ is limited by the flow resulting from the coalescence. A dimensional analysis of the Navier-Stokes equations in the viscous flow steadystate limit (constant $d L_{m} / d t$ ) gives [5] $\nabla P=\eta \Delta U$, where $P=\sigma / L_{m}$ is the capillary pressure and $\eta$ is the shear viscosity. Viscosity remains nearly constant in the vicinity of the critical point. Its value is taken as $\eta \approx 2.7 \times$ $10^{-6} \mathrm{~Pa} \cdot \mathrm{s}$ as obtained from an extrapolation at $T_{c}$ of the data from Ref. [6]. Liquid-vapor interfacial tension $\sigma$ obeys the power law behavior $\sigma=\sigma_{0} \tau^{2 \nu}$ with respect to the reduced temperature $\tau=\left(T_{c}-T\right) / T_{c}$, where $T$ and $T_{c}$ (in $\mathrm{K}$ ) represent temperature and critical temperature, respectively. $\nu=0.63$ is the universal exponent; $\sigma_{0}=5.7 \times$ $10^{-3} \mathrm{~N} \cdot \mathrm{m}^{-1}[4]$ is a critical amplitude that is system dependent. As $L_{m}$ is the typical length scale, $\nabla P=\eta \Delta U$ can be written as $\sigma / L_{m}^{2}=\left(\eta / L_{m}^{2}\right)\left(d L_{m} / d t\right) . L_{m}$ follows the linear growth law given by

$$
L_{m}=b(\sigma / \eta) t
$$

where $b \approx 0.03$ is a universal constant, independent of the fluid properties and $\sigma / \eta$ is a known function of $\tau$.

Experimental. - The large diamagnetic susceptibility of $\mathrm{H}_{2}$ enables gravity forces to be compensated by the magnetic field $\left(B_{0}\right)$ gradient such as $B_{0} \nabla B_{0} \approx 491 \mathrm{~T}^{2} \mathrm{~m}^{-1}$ [4]. This value is provided near the end of a superconductive coil. The cell containing the fluid is cylindrical $(3 \mathrm{~mm}$ in diameter) with its axis horizontal, perpendicular to the coil axis. It is made of sapphire and is closed by two parallel 
sapphire windows $3 \mathrm{~mm}$ apart and sealed by indium rings. The gravity forces are compensated at a unique point. In such a small sample, however, the residual forces, which are directed towards the coil axis and are proportional to the distance to it, give a contribution lower than $1.5 \times$ $10^{-2} \mathrm{~g}$ (here $\mathrm{g}$ is the Earth acceleration value). The cell is vibrated sinusoidally by means of an electric stepper motor equipped with an adjustable eccentricity cam (amplitude range $<0.5 \mathrm{~mm}$, frequency range $<50 \mathrm{~s}^{-1}$ ). The use of sapphire prevents heating by eddy currents when the sample is under vibrations. A parallel beam illuminates the sample, which is observed with a CCD camera. The cell is filled with a capillary that can be sealed with an ice floe. The cell is held in a thermostat with $0.3 \mathrm{mK}$ long term (hour) accuracy without vibration. Accuracy is 10 times better on a short time period (minute). Temperature can be varied between 9 and $40 \mathrm{~K}$, and pressure up to $2 \mathrm{MPa}$. The temperature quenches are very fast (on the order of a few ms) as sapphire exhibits an increase in thermal conductivity around $30 \mathrm{~K}$. A difficulty in such vibrated experiments is the temperature control. When the vibration is set, magnetic-induced currents in the temperature sensor and its connecting wires prevent accurate temperature measurements and temperature control. Hence we adopted a procedure where temperature was regulated by sending a constant power and thermal quench initiated by lowering this power. The phase separation process is always very fast, lasting between 0.5 to $15 \mathrm{~s}$ (Figs. 1 and 2).

The cell is filled to critical density. When $\mathrm{H}_{2}$ is cooled in the cell, the ortho- $\mathrm{H}_{2}$-para- $\mathrm{H}_{2}$ equilibrium is shifted and the percentage of para- $\mathrm{H}_{2}$ increases from $25 \%$ at room temperature to $96 \%$ at $30 \mathrm{~K}$ [7], with a time constant on the order of $50 \mathrm{~h}$. The critical coordinates of $p-\mathrm{H}_{2}$ are $T_{c}=$ $32.976 \mathrm{~K}, P_{c}=1.2928 \mathrm{MPa}$, and $\rho_{c}=31.426 \mathrm{~kg} \cdot \mathrm{m}^{-3}$ [7].

The typical wavelength of the pattern (see Fig. 1) is analyzed by measuring, in several directions, the spatial frequency of the domains directly on the pictures. The resolution is on the order of $20 \mu \mathrm{m}$. Twenty-seven experimental runs were performed with vibrations in the frequency range 5-50 s${ }^{-1}$, amplitude range 53-500 $\mu \mathrm{m}$, and $T_{c}-T$ range $0.08-1.1 \mathrm{mK}$.

Phase transition. - A typical evolution pattern at near critical density $(\phi>0.3)$ is shown in Fig. 1 together with the Fourier transform of the patterns. As shown in Figs. 1(a) and 1(b), the domains are interconnected and the pattern remains isotropic during the experiment time. There is a noticeable exception for the highest values of frequency and amplitude $\left[a \geq 0.4 \mathrm{~mm}, f \geq 40 \mathrm{~s}^{-1}\right.$; see Fig. 1(c), case denoted as (c)]. Here, at late times, the domains grow more rapidly perpendicular to the vibration direction and become anisotropic.

Without vibration, the evolution of domain wavelength $L_{m}$ is proportional to time, with slope $d L_{m} / d t=U$. A typical evolution (corresponding to $T_{c}-T=0.13 \mathrm{mK}$ ) is plotted in the inset of Fig. 2. Under vibration, the pattern wavelength evolution is more complicated, as reported in

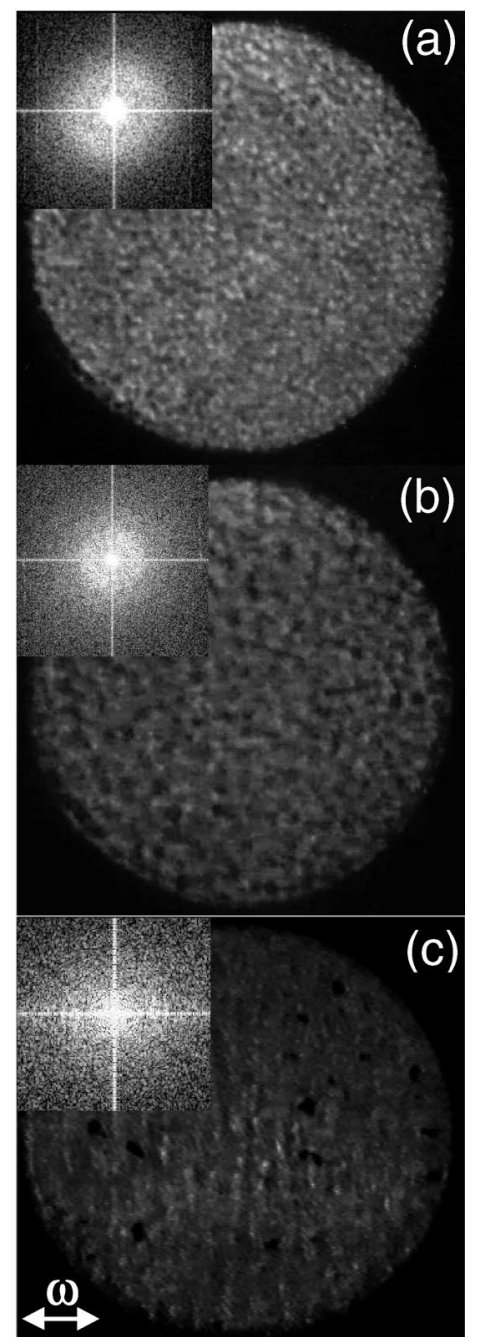

FIG. 1. Phase transition in $\mathrm{H}_{2}$ under vibration: gas-liquid interconnected pattern after a quench of about $0.6 \mathrm{mK}$ below $T_{c}$. The insets correspond to the Fourier transforms of the image. (a) Vibration of $0.3 \mathrm{~mm}$ amplitude and $20.3 \mathrm{~s}^{-1}$ frequency, $0.6 \mathrm{~s}$ after quench; (b) the same vibration, $1.7 \mathrm{~s}$ after quench; (c) vibration of $0.53 \mathrm{~mm}$ amplitude and $40 \mathrm{~s}^{-1}$ frequency, $3.6 \mathrm{~s}$ after quench.

Fig. 2 for a typical evolution under vibration at $T_{c}-T=$ $0.6 \mathrm{mK}$ : (a) For small $L_{m}$, a linear growth similar to the evolution without vibration is observed [Figs. 1(a) and 2; linear evolution denoted as (a)]. (b) When $L_{m}$ exceeds a crossover length $L_{0}$, another linear growth law is observed [Figs. 1(b) and 2, linear evolution denoted as (b)], with larger slope $d L_{m} / d t=U^{*}$ corresponding to a growth acceleration. Because of the limitations on optical resolution, this crossover is visible only for vibrations with a large enough frequency and amplitude (roughly corresponding to $a \omega>10 \mathrm{~mm} \cdot \mathrm{s}^{-1}$ ).

Analysis. - Even though the temperature cannot be accurately measured for each quench, the domain evolution without vibration is well known. We therefore use Eq. (1) to infer the $\tau$ value from the $U(\tau)$ dependence, $\tau=\left[\eta /\left(b \sigma_{0}\right)\right]^{1 /(2 \nu)} U^{1 / 2 \nu} \sim U^{0.79}$. 


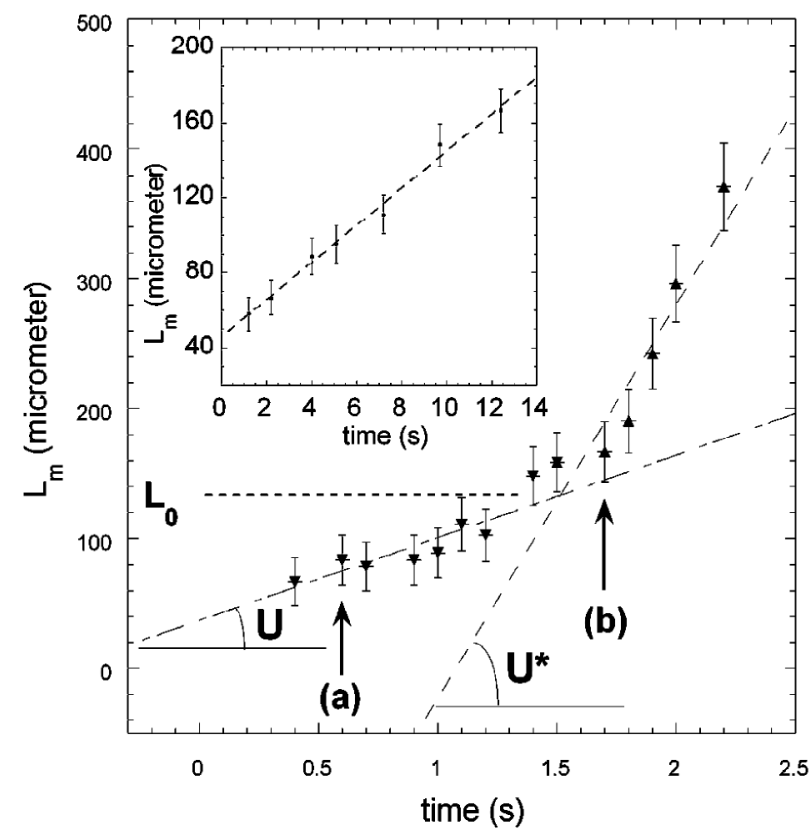

FIG. 2. Typical evolution of the pseudowavelength $L_{m}$ between interconnected phase separating domains (vibration $a=$ $0.3 \mathrm{~mm}, f=20.3 \mathrm{~s}^{-1}$ ). $U$ and $U^{*}$ are growth velocities that are functions of $T_{c}-T$. The evolution for $L_{m}<L_{0}$ corresponds to $T_{c}-T=0.6 \mathrm{mK}$. The inset shows typical evolution without vibrations at $T_{c}-T=0.13 \mathrm{mK}$. The arrows correspond to Figs. 1(a) and 1(b).

Incompressible fluids under vibration [8] display different regimes dominated by either viscous or inertial effects. The key parameter to determine the different regimes is the thickness $\delta$ of the viscous boundary layer

$$
\delta=(2 \mu / \omega)^{1 / 2},
$$

where $\mu=\eta / \rho$ is the kinematic viscosity. When $L_{m} \ll$ $\delta$, the two phases strongly interact by viscous shear and move coherently. The growth is thus unaffected by vibrations. However, as soon as $L_{m}>\delta$, the motion of the two phases are locally uncoupled such that they no longer exhibit the same velocity due to inertial effect. At large $L_{m}$, the crossover length $L_{0}$ that separates the two growth regimes should then correspond to the thickness of the viscous boundary layer, $L_{0} \sim \delta$. In Fig. 3, we have plotted $L_{0}$ with respect to $\omega$ in a $\log -\log$ plot. A fit to $L_{0}=l_{0} \omega^{-x}$ gives $x=0.54 \pm 0.07$, a value in good agreement with Eq. (2). The fit with $x=1 / 2$ imposed gives $l_{0}=1660 \pm$ $50 \mu \mathrm{m} \cdot \mathrm{s}^{-1 / 2}$, which can be compared to $420 \mu \mathrm{m} \cdot \mathrm{s}^{-1 / 2}$ obtained from Eq. (2) assuming $L_{0}=\delta$. The factor of 4 difference between the amplitudes is not significant at this stage of the analysis and therefore the region $L_{m}<L_{0} \sim \delta$ can be taken to be the region where vibrations do not affect the growth. We thus can take advantage of the undisturbed variation of $L_{m}$ with $t$ at $L_{m}<\delta$ to determine the reduced temperature $\tau$ from velocity $U$.

The acceleration of the growth in the region $L_{m}<\delta$ is then due to the presence of an inertial velocity difference

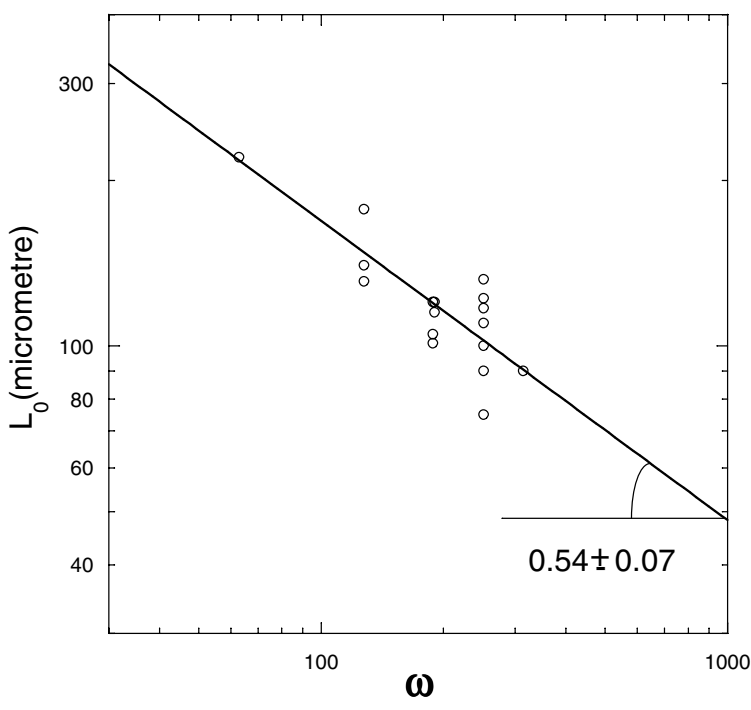

FIG. 3. Variation of the crossover length $L_{0}$ (see Fig. 2) with respect to the angular frequency $\omega(\log -\log$ plot $)$. The line is the best fit to a power law.

$\Delta V \sin \omega t$, between gas and liquid domains that scales as

$$
\Delta V \sim\left(\Delta \rho / \rho_{c}\right) a \omega .
$$

The gas-liquid density difference is $\Delta \rho=\rho_{1}-\rho_{\mathrm{g}}$, where $\rho_{\mathrm{g}}, \rho_{1}$ are the gas and liquid densities, respectively. In other words, Eq. (3) means that the domains are displaced on about $\left(\Delta \rho / \rho_{c}\right) a$ during a vibration period.

In order to evaluate the relative influence of viscous and inertial flows at the domain length scale, it is necessary to evaluate the Reynolds number $\mathrm{Re}=U L_{m} / \mu$. From Eqs. (2) and (3), $\operatorname{Re}=2(\Delta \rho / \rho)\left(a L_{m} / \delta^{2}\right)$. In the critical region [9], $\Delta \rho=\rho_{1}-\rho_{\mathrm{g}}=2 \rho_{c} B \tau^{\beta}$, where $\rho_{c}$ is the critical density, $B$ (=1.61 from Ref. [10]) is a critical amplitude and $\beta=0.325$ is the universal exponent. The evaluation with Fig. 2(b) data gives a typical value $\operatorname{Re}>$ 1.5 for $L_{m}>\delta$. Thus vibration-induced inertial effects, which increase with the evolution of $L_{m}$, progressively overwhelm viscosity effects, in agreement with the previous analyses.

In the following analysis, we consider only dimensional and scaling arguments. In the problem, there are only three length scales, $L_{m}, \delta$, and $(\Delta \rho / \rho) a$, the vibration amplitude of the domains. Numerical estimation of $(\Delta \rho / \rho) a$ [e.g., by using the typical data in Figs. 1(a) and 1(b)] shows that this length is always much smaller than the range of studied $L_{m}$. We thus restrain our analysis to the comparison of $L_{m}$ with $\delta$. Three main regimes can be identified. (a) $L_{m}<\delta$, where viscous effects are predominant. As discussed above, the gas and liquid phases move coherently and growth is unaffected by vibrations. This stage corresponds to Fig. 1(a) and case (a) of Fig. 2. (b) $L_{m}>\delta$ [Fig. 1(b) and case (b) of Fig. 2]. A velocity gradient appears at the domain scale; shear flow stretches the domains. (c) $L_{m} \gg \delta$. The domains order in the direction perpendicular to the vibration direction [Fig. 1(c)]. Other mechanisms than 
shear flow, as, e.g., the Bernoulli pressure difference $\Delta P$, directed perpendicular to the vibration direction and which scales as $\Delta P \sim \Delta V$, can deform the domain interface and enhance domain coalescence in the direction perpendicular to vibration. A detailed analysis of this process, however, is well beyond the scope of this study, devoted to regime (b).

In this latter regime where $L_{m}>\delta$, one can first try to account for growth acceleration by using dimensional considerations. The growth velocity increases from $U$ to $U^{*}$ (as defined in Fig. 2) proportional to $\Delta V$, leading to

$$
\Delta U_{G}=U^{*}-U \sim \Delta V \sim\left(\Delta \rho / \rho_{c}\right) a \omega .
$$

This relation implies hydrodynamic nonlinearities and does not provide any details on the mechanisms about the origin of the growth acceleration.

Another possibility is the influence of the periodic shear flow of amplitude $S \approx \Delta V / \delta$ that develops between the domains. The shear amplitude is on the order of $100 \mathrm{~s}^{-1}$ [using, e.g., the Fig. 1(b) data and Eqs. (2) and (3)]. In shear flow processes, the important parameter is the dimensionless product of shear with typical time $[3,11]$. The typical time is the time when shear is able to deform the domains, here the vibration period $1 / f$. When this product is larger than unity [it is 5 when using the Fig. 1(b) data], one expects a noticeable effect of the shear on growth. The growth velocity increase $\Delta U_{S}=U^{*}-U$ can then be eventually written as

$$
\Delta U_{S} / U \sim S / f=2 \pi\left(\Delta \rho / \rho_{c}\right)(a / \delta) .
$$

The average deformation of domains is zero because the excitation is periodic; the pattern should then remain in average isotropic as observed in Fig. 1(b).

In both Eqs. (4) and (5), the proposed behaviors can be written in terms of experimental quantities as $\Delta U \sim$ $a^{x} \omega^{y} U^{z}$, with exponents $x, y, z$ that take the values $(1,1$, $0.26)$ from Eq. (4) and (1, 0.5, 1.26) from Eq. (5), respectively. In order to determine the set of best exponents, we construct the dimensionless function $C=\Delta U a^{-x} \times$ $\omega^{-y} U^{-z}$. This function should be a constant for all experimental values $(\Delta U, U, a, \omega)$ with the selection of the appropriate set of $x, y, z$ values. For this set, the relative error $s(C) /\langle C\rangle$, where $s(C)$ is the standard deviation and $\langle C\rangle$ the mean value, should be minimum. We varied $x, y, z$ in the range $[-2,+2]$ and report the result of the minimization of $s(C) /\langle C\rangle$ in Table I. A minimum is found at $s(C) /\langle C\rangle=0.6 \quad$ with $x=0.35 \pm 0.5, \quad y=0.35 \pm 0.5$, and $z=1.3 \pm 0.4$ (uncertainties correspond to 1 standard deviation). The values for $y$ and $z$ are close to the values of Eq. (5) (shear influence), but $x$ is somewhat too small. The sensitivity of the method is not very high, as one can see in Table I, which also reports the $s(C) /\langle C\rangle$ values when the exponents of Eqs. (4) and (5) are imposed. One notes, however, that the results of the shear model compare relatively well to the experimental data.

Concluding remarks. - Our investigation shows that the phase transition of a gas and a liquid in space under
TABLE I. Results of the minimization of the relative error $s(C) /\langle C\rangle$, where $C=\Delta U_{G, S} a^{-x} \omega^{-y} U^{-z}$.

\begin{tabular}{lcccc}
\hline \hline & $\frac{s(C)}{\langle C\rangle}$ & $\mathbf{x}$ & $\mathbf{y}$ & $\mathbf{z}$ \\
\hline Minimum & 0.60 & $0.35 \pm 0.5$ & $0.35 \pm 0.5$ & $1.3 \pm 0.4$ \\
$\Delta U_{G}$ [Eq. (4)] & 0.76 & 1 & 1 & 0.26 \\
$\Delta U_{S}$ [Eq. (5)] & 0.75 & 1 & 0.5 & 1.26 \\
\hline \hline
\end{tabular}

weightlessness conditions can be significantly accelerated by high-frequency vibrations. The vibrations initiate velocity differences between the gas-liquid growing domains, whereby the domain size grows larger than the viscous boundary layer. Across the domains, shear flow and Bernoulli pressure difference participate to enhance domain coalescence and speed up growth. Domains eventually become anisotropic and order in periodic planes perpendicular to the vibration direction. These hydrodynamics phenomena that couple with phase transition reveal intriguing new processes. Their precise theoretical description is still lacking and it is expected that the present experimental investigation will foster further examination.

We thank V. Nikolayev for fruitful discussions and C. Erkey for a critical reading of the manuscript. This work has been partially supported by the Centre National d'Etudes Spatiales.

[1] D. Beysens and Y. Garrabos, Physica (Amsterdam) 281A, 361 (2000), and references therein.

[2] G.Z. Gershuni and D. V. Lyubimov, Thermal Vibrational Convection (John Wiley \& Sons, Chichester, 1998).

[3] A. Onuki, Phase Transition Dynamics (Cambridge University Press, Cambridge, 2002).

[4] R. Wunenburger, D. Chatain, Y. Garrabos, and D. Beysens, Phys. Rev. E 62, 469 (2000).

[5] E. D. Siggia, Phys. Rev. A 20, 595 (1979).

[6] D. E. Diller, J. Chem. Phys. 42, 2089 (1965).

[7] Encyclopédie des gaz de L'Air Liquide (Elsevier, Paris, 1976), p. 915.

[8] L.D. Landau and E.M. Lifshitz, Fluid Mechanics (Pergamon Press, Elmsford, NY, 1975).

[9] H.E. Stanley, Introduction to Phase Transitions and Critical Point Phenomena (Oxford University Press, Oxford, 1971).

[10] M. R. Moldover, Phys. Rev. A 31, 1022 (1985).

[11] The effect on phase transition of a $600 \mathrm{~s}^{-1}$ oscillating shear flow has already been investigated in a liquid mixture near its liquid-liquid critical point [12]. A steady state of partial phase separation was obtained, corresponding to the balance between shear homogenization and phase separation. The domains were highly anisotropic, stretched in the flow direction. In these experiments, the flow was produced in a rectangular pipe and the shear was nearly spatially uniform in the sample, which makes important differences with the present study where shear exhibits a spatial pseudoperiod $L_{m}$ and frequency was much larger than in [12] where $f=[0.03-0.3] \mathrm{s}^{-1}$.

[12] D. Beysens and F. Perrot, J. Phys. Lett. 45, L31 (1984). 